\title{
Dois milhões de casos da COVID-19 no Brasil
}

\author{
Two million COVID-19 cases in Brazil \\ Claudio José dos Santos Júnior ${ }^{1}$ (D), Thiago José Matos Rocha ${ }^{1}$
}

\section{RESUMO}

Em 11 de março de 2020, a Organização Mundial da Saúde (OMS) declarou oficialmente a pandemia da doença coronavírus 2019 (COVID-19), uma doença respiratória aguda, causada por um novo coronavírus (SARS-CoV-2, anteriormente conhecido como 2019-nCoV). A COVID-19 se espalhou por toda a China e recebeu atenção mundial. Este trabalho chama atenção para o impacto da marca histórica de 2 milhões de casos confirmados da COVID-19 no Brasil e para o fato de o país ser o segundo do mundo com mais mortes pela doença em números absolutos. Destacamos que o afrouxamento das estratégias de isolamento social e domiciliar surgem no Brasil justamente no período em que se revela a expectativa de o país virar o novo epicentro da pandemia.

Palavras-chave: COVID-19; Doença do novo coronavírus 2019; 2019-nCoV; Coronavírus; SARS-CoV-2.

\begin{abstract}
On March 11, 2020, the World Health Organization (WHO) officially declared the 2019 coronavirus disease (COVID-19), an acute respiratory disease caused by a new coronavirus (SARS-CoV-2, previously known such as 2019-nCoV), as a pandemic. COVID-19 has spread across China and received worldwide attention. Here, we draw attention to the impact of the historical mark of 2 million cases confirmed by COVID-19 in Brazil and to the fact that, in absolute numbers, Brazil is the second in the world with more deaths from COVID-19. We discuss the fact that, in a moment in which Brazil seems to become the new epicenter of the COVID-19 pandemic, it has been implemented strategies for relaxing social distancing and social isolation.
\end{abstract}

Keywords: COVID-19; 2019 novel coronavirus; 2019-nCoV; Coronavirus; SARS-CoV-2.

1. Universidade Estadual de Ciências da Saúde de Alagoas

$\triangle$ Claudio José dos Santos Júnior. Faculdade de Medicina (FAMED-UNCISAL). Rua Dr. Jorge de Lima, 113 - Trapiche da Barra. CEP: 57010-300. Maceió (AL), Brasil. claudiosantos_al@hotmail.com | Recebido em: 13/06/2020 | Aprovado em: 17/07/2020 
O surto de uma "pneumonia atípica", posteriormente nominada de Doença do Coronavírus 2019 (COVID-19), em Wuhan, na China, constituiu o terceiro de um vírus da família dos coronavírus humano (HCoVs) nos últimos 20 anos. O primeiro foi de síndrome respiratória aguda grave (SARS-CoV), com início também na China em 2002, e o segundo, responsável pela síndrome respiratória do Oriente Médio (MERS-CoV), datado de 2012, com início na Arábia Saudita ${ }^{1}$.

A declaração da Organização Mundial da Saúde (OMS) da infecção pelo SARS-CoV-2 como Emergência de Saúde Pública de Interesse Internacional, em 30 de janeiro de 2020, com posterior anúncio, em 11 de março de 2020, de Pandemia de COVID-19, surge devido às altas taxas de mortalidade de casos na China, ao seu rápido potencial de disseminação para outras nações e a previsão do importante impacto econômico-global da patologia sobre os sistemas de saúde dos países ${ }^{2}$.

Desse período até os dias atuais, tem-se revelado, através da pesquisa clínica e pré-clínica, que o SARS-CoV-2 (anteriormente nominado de 2019nCoV) possui tempo de incubação de 3 a 7 dias, podendo se alongar até 14 dias $^{3}$, e que, embora apresente semelhanças filogenéticas com outros HCoVs, sua transmissibilidade é muito maior e, quando comparada ao SARS-CoV, é dez vezes mais rápida4.

O quadro clínico da COVID-19 é, em sua maioria, leve $(80 \%)$ e semelhante ao de outras viroses respiratórias, quais sejam: febre, tosse seca, fadiga e, em casos mais severos (5-14\%), dispnéia, hemoptise, linfopenia importante e insuficiência renal aguda (IRA) $)^{5,6}$. Alguns trabalhos sugerem que o bloqueio da expressão da Enzima Conversora de Angiotensina 2 (ECA2), presente em grandes quantidades em células do coração e do pulmão, predispõem a miocardite e a insuficiência respiratória ${ }^{7,8}$. Febre e disfunção de múltiplos órgãos são igualmente relacionadas à capacidade da infecção viral de produzir uma reação imune excessiva no hospedeiro, uma 'tempestade de citocinas', principalmente de interleucina 6 (IL-6), gerando extenso dano tecidual ${ }^{9}$. Dessa forma, a produção aumentada de citocinas pró-inflamatórias levaria o organismo a uma resposta inflamatória descompensada e ao estado de Síndrome da Resposta Inflamatória Sistêmica (SIRS).

O diagnóstico dos casos sintomáticos é confirmado com a pesquisa do SARS-CoV2 por biologia molecular (RT-PCR em tempo real) em aspirado nasal ou por método imunológico através de sorologias clássicas para detecção de anticorpos IgG ou IgM. No entanto, no Brasil, a falta de testes para identificar novos infectados faz com que muitos casos da doença não sejam diagnosticados, visto que a testagem é realizada em populações selecionadas. Inicialmente, o Ministério da Saúde recomendou a testagem apenas dos casos de pacientes internados com quadro respiratório grave e, somente mais recentemente, mudou seu protocolo, passando todos os casos de síndrome gripal ${ }^{10,11}$.

Não há, até o momento, tratamento antiviral específico recomendado para a COVID-19. Do mesmo modo, ainda não existe uma vacina disponível no mercado. Enquanto os esforços para o desenvolvimento de um imunobiológico seguro e eficaz ainda não foram concluídos, outros avanços foram conseguidos, como resultados positivos da aplicação de imunobiológicos contra a Covid-19, que mostraram produzir anticorpos neutralizadores do SARS-CoV2 semelhantes aos encontrados em pacientes recuperados. Esses resultados promissores fizeram com que fosse iniciada, inclusive no Brasil, a fase 3 dos testes de uma vacina contra a COVID-19, última etapa da pesquisa antes da obtenção do registro sanitário, que tem por objetivo demonstrar a sua eficácia em seres humanos ${ }^{12}$.

O tratamento sintomático e de suporte, associado a oxigenoterapia, têm sido relatadas como as principais intervenções terapêuticas recomendas. Recentemente, um estudo conduzido no Reino Unido pela Universidade de Oxford identificou a primeira droga capaz de reduzir mortes pela COVID-19 em indivíduos com quadros graves. Os números da pesquisa mostraram uma redução da mortalidade de $35 \%$ em pacientes com COVID-19 que precisavam de respiradores e de $20 \%$ no grupo que necessitou de suporte de $\mathrm{O}_{2}$ que fizeram uso de dexametasona $6 \mathrm{mg} / \mathrm{dia}$ por 10 dias $^{13}$. A ventilação mecânica pode ser necessária em casos de insuficiência respiratória refratária à oxigenoterapia ${ }^{9}$, entretanto, no Brasil, as técnicas de ventilação mecânica não-invasiva (VNI) e de uso de cateter nasal de alto fluxo (CNAF) vêm sendo proscritas devido à alta capacidade infectante do SARS-CoV2 ${ }^{14}$. Medidas gerais como uso de máscara, higienização das mãos e isolamento domiciliar de pacientes com sintomas gripais vêm sendo sugeridas como estratégia de prevenção $0^{2,9}$. 
No surto de Vírus Ebola, em 2015, um estudo internacional demonstrou que medidas de intervenção não-farmacêuticas podem, ao mesmo tempo, reduzir a mortalidade da pandemia e serem economicamente benéficas ${ }^{16}$. No âmbito nacional, entretanto, o afrouxamento das estratégias de isolamento social e domiciliar surgem justamente no período em que se revela a expectativa de o país virar o novo epicentro da pandemia ${ }^{15}$ e após o Brasil alcançar a marca histórica de 2 milhões de casos confirmados da COVID-19. O país é, ainda, o segundo do mundo com maior número absoluto de mortos pela doença ${ }^{17}$.

A COVID-19 tem pressionando a comunidade científica nacional e internacional a desenvolverem, rapidamente, um conjunto de estratégias terapêuticas e de profilaxia para conter o avanço do SARS-CoV2. Porém, enquanto uma estratégia duradoura de profilaxia ainda não foi finalizada, espera-se que os comportamentos individuais e coletivos e que as ações do Estado sigam as principais evidências científicas que apontam as medidas de distanciamento social como única estratégia disponível no momento, capaz de mitigar a pandemia e suas perdas.

Em concordância com o médico e escritor americano, Oliver Wendell Holmes, "O mais importante da vida não é a situação em que estamos, mas a direção para a qual nos movemos", resta-nos ponderar, aqui, os perigos do afrouxamento das estratégias de isolamento social neste momento e a certeza de que a Doença do Coronavírus 2019 é um dos maiores senão o maior desafio a ser enfrentado dos últimos tempos, carecendo que as estratégias nacionais de enfrentamento dessa importante problemática de saúde sejam conduzidas com responsabilidade pelos gestores públicos e pela sociedade em geral.

\section{REFERÊNCIAS:}

1. Rothan HA, Byrareddy SN. The epidemiology and pathogenesis of coronavirus disease (COVID-19) outbreak. Journal of Autoimmunity. 2020:102433-102433. https://doi.org/10.1016/j.jaut.2020.102433.

2. Purcell LN, Charles AG. An Invited Commentary on "World Health Organization declares global emergency: A review of the 2019 novel Coronavirus (COVID-19)": Emergency or new reality? International Journal of Surgery. 2020, 76: 111. https://doi.org/10.1016/j. ijsu.2020.03.002.

3. Centers for Disease Control and Prevention (CDC). The Novel Coronavirus Pneumonia Emergency Response
Epidemiology Team. The epidemiological characteristics of an outbreak of 2019 novel coronavirus diseases (COVID-19) in China. Zhonghua Liu Xing Bing Xue Za Zhi. 2020;41(2):145-51. China, 202. China CDC Weekly.2020,2(8):113-122.

4. Mizumoto K, Kagaya K, Zarebski A, Chowell G. Estimating the asymptomatic proportion of coronavirus disease 2019 (COVID-19) cases on board the Diamond Princess cruise ship, Yokohama, Japan, 2020. Euro Surveill. 2020;25(10):2000180.

5. Khachfe H H, Chahrour M, Sammouri J, Salhab H, B Makki BE, Fares M. An Epidemiological Study on COVID-19: A Rapidly Spreading Disease. Cureus 12(3):e7313. 2020. https://doi.org/10.7759/cureus.7313.

6. Chen N, Zhou M, Dong X, Qu J, Gong F, Han Y, et al. Epidemiological and clinical characteristics of $99 \mathrm{ca}-$ ses of 2019 novel coronavirus pneumonia in Wuhan, China: a descriptive study. Lancet (London, England). 2020;395(10223):507-13.https://doi.org/10.1016/ S0140-6736(20)30211-7.

7. Xiong TY, Redwood S, Prendergast B, Chen M. Coronaviruses and the cardiovascular system: acute and long-term implications. Eur Heart J. 2020.

8. Zheng YY, Ma YT, Zhang JY, Xie X. COVID-19 and the cardiovascular system. Nat Rev Cardiol. 2020. https:// doi.org/10.1038/s41569-020-0360-5.

9. Cascella M, Rajnik M, Cuomo A; Dulebohn SC; Di Napoli R. Features, Evaluation and Treatment Coronavirus (COVID-19). Stat Pearls Publishing, Treasure Island, FL; 2020.

10. Li Z, Yi Y, Luo X, Xiong N, Liu Y, Li S, et al. Development and Clinical Application of A Rapid IgM-IgG Combined Antibody Test for SARS-CoV2 Infection Diagnosis. J Med Virol. 2020. https://doi.org/10.1002/jmv.25727.

11. Guo L, Ren L, Yang S, Xiao M, Chang D, Yang F, et al. Profiling Early Humoral Response to Diagnose Novel Coronavirus Disease (COVID-19). Clin Infect Dis. 2020. https://doi.org/10.1093/cid/ciaa310.

12. Organização Mundial da Saúde, Draft landscape of Covid-19 candidate vaccines. [Acesso em 28 de junho de 2020]. Disponível em: https://www.who.int/who-documents-detail/ draft-landscape-of-covid-19-candidate-vaccines.

13. Recovery. Randomised Evaluation of Covid-19 Therapy. [Acesso em 28 de junho de 2020]. Disponível em: https://www.recoverytrial.net/results

14. Associação de Medicina Intensiva Brasileira. Manuseio do paciente com infecção pelo Coronavírus COVID-19 e pneumonia e insuficiência respiratória. Comitê de Ventilação Mecânica da AMIB. Brasil: Comitê de Ventilação Mecânica da AMIB; 2020.

15. Menezes PL, Garner DM, Valenti VE. Brazil is projected to be the next global Covid-19 pandemic epicenter. MedRxiv 2020. https://doi.org/10.1101/2020.04.28.20083675

16. Pellecchia U, Crestani R, Decroo T, et al. Social Consequences of Ebola Containment Measures in Liberia. PLoS ONE. 2015;10(12):e0143036.

17. COVID-19. Dashboard by the Center for Systems Science and Engineering (CSSE) at Johns Hopkins University (JHU) [Acesso em 17 de julho de 2020]. Disponível em: https://coronavirus.jhu.edu/map.html. 